Journal of New Results in Science
$\mathrm{https} / /$ dergipark.org.tr/en/pub/jnrs
Research Article
Open Access

\title{
A study on radiation shielding potentials of green and red clayey soils in Turkey reinforced with marble dust and waste tire
}

\author{
Keywords: \\ Green and red clayey \\ soil, \\ Radiation shielding, \\ EpiXS, \\ Marble dust, \\ Waste tire
}

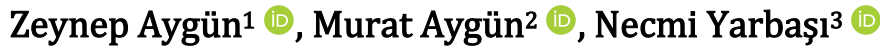

\begin{abstract}
The increasing radiation applications in our daily life makes it essential to protect ourselves from the harms of radiation by using alternative, cheap and natural materials. The present study aimed to analyze the radiation shielding abilities of green and red clayey soils from Oltu/Erzurum in Turkey, reinforced with waste tires and marble dust. For the purpose to investigate the shielding features of the samples, radiation attenuation parameters were determined by using EpiXS software, which can calculate partial or total cross-sections, partial or total mass attenuation coefficients, electron densities, effective atomic numbers, and buildup factors for energy absorption and exposure between $1 \mathrm{keV}$ and $1 \mathrm{GeV}$. We compared the obtained mass attenuation coefficients and total atomic cross-section values of the samples with those of a widely used shielding material, ordinary concrete, to make a meaningful evaluation about the shielding potentials of the samples. To validate obtained values by EpiXS, we also calculated the mass attenuation coefficients of the samples by XCOM code, and compatible results were obtained. Among all the studied clayey soil samples, green clay reinforced with marble dust and waste tire has the highest shielding capability. It can also be mentioned that reinforcement with marble dust and waste tire improves the shielding ability of the clayey soils.
\end{abstract}

Subject Classification (2020):

\section{Introduction}

Soil materials such as clayey soils are essential in geologic, construction, and environmental applications. Soil performances for some purposes can be improved by reinforcing with natural resources and types of waste based on their environment-friendly and cost-effectiveness aspects. Marble dust (MD) and waste tire (WT) were commonly chosen for reinforcing the soil materials and attracts the attention of researchers for different purposes [1-4]. Reusing waste materials is also important and can make it possible to reduce environmental problems. However, these kinds of natural and cheap materials are also significant for radiation shielding. Radiation is widely used in energy production (nuclear reactors), space exploration, medical imaging and treatment, material investigations, archaeology, military etc. [5]. Due to the increase of radiation applications in daily lives, protection from the harms of radiation has become more important nowadays. It is also important to

\footnotetext{
${ }^{1}$ zeynepyarbasi@ hotmail.com (Corresponding author); ${ }^{2}$ maygun @ beu.edu.tr; ${ }^{3}$ nyarbasi@ atauni.edu.tr

${ }^{1}$ Vocational School of Technical Sciences, Bitlis Eren University, Bitlis, Turkey

${ }^{2}$ Department of Physics, Faculty of Science and Arts, Bitlis Eren University, Bitlis, Turkey

${ }^{3}$ Department of Geotechnics, Faculty of Engineering, Atatürk University, Erzurum, Turkey

Article History: Received: 23 Aug 2021 — Accepted: 05 Oct 2021 — Published: 31 Dec 2021
} 
use alternative materials as building materials for shielding purposes. There are many studies about these kinds of materials in the literature [6-11]. Clay is one of the eco-friendly and cost-effective materials which can be used as a shielding material in construction and building purposes in many nations [11]. Important knowledge about the shielding properties of the materials can be obtained by determining radiation attenuation parameters. Radiation interaction coefficients of materials can be calculated by widely used codes such as XCOM [12], GEANT4 [13], WinXCom [14,15], and XMuDat [16], and recently Phy-X/PSD [17] and Py-MLBUF [18]. Among the lately reported codes, EpiXS makes it possible to determine the radiation shielding parameters such as mass attenuation coefficient, effective atomic number, electron density, atomic cross-section and buildup factors without knowing the density [19]. This feature takes the program one step ahead in terms of usability. Several research is done by using EpiXS recently [20-23].

The present study aimed to investigate the photon attenuation parameters; mass attenuation coefficients (MAC), effective atomic number $\left(Z_{e f f}\right)$, electron density $\left(N_{e f f}\right)$, total atomic cross-section (ACS) and buildup factors of green clayey (GC) and red clayey (RC) soils reinforced with WT and MD to learn the radiation shielding potentials of the samples. In this regard, we used EpiXS code which can perform in the energy range of $1 \mathrm{keV}-1 \mathrm{GeV}$. The studied clay materials show the characteristics of the Oltu/Erzurum region consisting of Oligocene lower upper sedimentary units, volcanic rocks and upper sedimentary units. The lower sedimentary unit consists of silt-clay layers, conglomerate sandstone, and gypsum-limestone band. The upper sedimentary unit has high clay content $[1,24]$. To the best of our knowledge, there is no paper about the shielding potentials of the used materials in the literature. By this investigation, the determination of radiation-matter interaction parameters of unreinforced and reinforced (with MD and WT) clayey soils from Oltu/Erzurum region can contribute to the literature.

\section{Materials and Methods}

\subsection{Samples}

In this study, the used samples were taken from literature reported by Aygun and Yarbasi [1]. GC and $\mathrm{RC}$ samples were obtained from Oltu (Erzurum) in Turkey. WT fragments were obtained from the Erzurum industry region. Obtained shredded waste tire pieces were shaken in the sieve machine. MD was provided from the Afyon region (Turkey) by polishing, scraping, and carving the marbles. The clay samples were also prepared by reinforcing with 5\% MD and 5\% MD with 0.5\% WT.

\subsection{Calculation Process}

The MAC, a quantity that defines the interaction possibility between incident photons and the mass per unit area, can be calculated by the Beer-Lambert formulated as:

$$
\begin{gathered}
I=I_{0} e^{-\mu t} \\
\mu_{m}=\frac{\mu}{\rho}=\ln \left(I_{0} / I\right) / \rho t=\ln \left(I_{0} / I\right) / t_{m}
\end{gathered}
$$

where $I_{0}$ and $I$ are incidents and attenuated photon intensities, $\rho\left(\mathrm{g} / \mathrm{cm}^{3}\right)$ is the density of a material, $\mu_{m}\left(\mathrm{~cm}^{2} / \mathrm{g}\right)$ and $\mu\left(\mathrm{cm}^{-1}\right)$ are mass, and linear attenuation coefficients, $t_{m}\left(\mathrm{~g} / \mathrm{cm}^{2}\right)$ and $t(\mathrm{~cm})$ are sample mass thickness (the mass per unit area) and the thickness, respectively.

We can write the total MAC for any compound as follows [25];

$$
\mu / \rho=\sum_{i} w_{i}(\mu / \rho)_{i}
$$

where $w_{i}$ and $(\mu / \rho)_{i}$ are the weight fraction and the MAC of the ith constituent element, respectively. $\operatorname{ACS}\left(\sigma_{T}\right)$ is defined as the sum of partial cross-sections in Eq. 2.4, 


$$
\sigma_{T}=\sigma_{P E}+\sigma_{\text {coh }}+\sigma_{\text {incoh }}+\sigma_{P P-N}+\sigma_{P P-E}
$$

where $\sigma_{\mathrm{PE}}, \sigma_{\mathrm{coh}}, \sigma_{\text {incoh}}, \sigma_{\mathrm{PP}-\mathrm{N}}$, and $\sigma_{\mathrm{PP} \text {-E }}$ are cross sections for photoelectric, coherent, incoherent, pair production in the nuclear field, and pair production in an electron field, respectively [19].

$Z_{\text {eff }}$ can be calculated by Eq. 2.5 where $\sigma_{e}$ is the electronic cross-section given by Eq. 2.6 [26]. $Z_{\text {eff }}$ can also be determined by an interpolation given in Eq. 2.7. In this equation, $\sigma_{1}$ and $\sigma_{2}$ are the elemental crosssections of two successive elements $Z_{1}$ and $Z_{2}$.

$$
\begin{gathered}
Z_{e f f}=\sigma_{T} \sigma_{e} \\
\sigma_{e}=\sum \frac{f_{i}}{Z_{i}}\left(\sigma_{T}\right)_{i} \\
Z_{e f f}=\frac{Z_{1}\left(\log \sigma_{2}-\log \sigma_{T}\right)+Z_{2}\left(\log \sigma_{T}-\log \sigma_{1}\right)}{\log \sigma_{2}-\log \sigma_{1}}
\end{gathered}
$$

$N_{\text {eff }}\left(\right.$ elctrs/g) parameter is directly proportional to its $Z_{\text {eff }}$ as given in Eq. 2.8 [27],

$$
N_{\text {eff }}=Z_{\text {eff }}\left(\frac{N_{A}}{\sum f_{i} A_{i}}\right)
$$

Energy absorption buildup factors (EABF) or exposure buildup factors (EBF) are calculated by the given formulas below [28,29]. G-P fitting parameters for the material can be calculated by using fitting parameters in the ANSI/ANS 6.4.3 [30] in Eq. 2.9 Buildup factors are calculated using Eq. 2.11 or 12 by determining $K(E, X)$ in Eq. 2.13. The distance from the source in $\mathrm{mfp}(\mathrm{cm})$ is given as $X$.

$$
\begin{gathered}
Z_{e q}=\frac{Z_{1}\left(\log R_{2}-\log R\right)+Z_{2}\left(\log R-\log R_{1}\right)}{\log R_{2}-\log R_{1}} \\
F=\frac{F_{1}\left(\log Z_{2}-\log Z_{e q}\right)+F_{2}\left(\log Z_{e q}-\log Z_{1}\right)}{\log Z_{2}-\log Z_{1}} \\
B(E, x)=1+\frac{(b-1)\left(K^{x}-1\right)}{(K-1)} \quad \text { for } K \neq 1 \\
B(E, x)=1+(b-1) x \quad \text { for } K=1 \\
K(E, x)=c x^{a}+d \frac{\tanh \left(\frac{x}{X_{k}}-2\right)-\tanh (-2)}{1-\tanh (-2)} \quad \text { for } \quad x \leq 40 \mathrm{mfp}
\end{gathered}
$$

\section{Results and discussion}

The chemical compositions of the used samples are given in Table 1 [1]. To validate the calculated values of unreinforced and reinforced clay materials by EpiXS, MAC values of the samples were also determined by XCOM [12], a well-known code, and a good agreement is obtained between the results.

Table 1. Chemical compositions (wt $\%$ ) of the studied samples.

\begin{tabular}{|c|c|c|c|c|c|c|c|c|c|c|c|c|c|c|}
\hline Samples & $\mathbf{0}$ & $\mathrm{Si}$ & $\mathrm{Al}$ & $\mathrm{Fe}$ & $\mathrm{Ca}$ & $\mathrm{Mg}$ & $\mathrm{Na}$ & $\mathrm{K}$ & $\mathrm{C}$ & $\mathrm{Ti}$ & $\mathrm{Mn}$ & $\mathrm{S}$ \\
\hline GC & 53.63 & 18.74 & 8.13 & 3.78 & 4.46 & 3.53 & 3.33 & 2.61 & 1.32 & 0.48 & - \\
\hline RC & 52.69 & 20.49 & 8.60 & 5.74 & 3.53 & 3.05 & 1.29 & 2.68 & 1.26 & 0.68 & - \\
\hline MD & 53.63 & 0.07 & - & - & 34.49 & 0.59 & - & - & 9.57 & 0.16 & - & 1.49 \\
\hline WT & 50.10 & 14.59 & 7.64 & 8.85 & 6.86 & 1.76 & 1.21 & 1.65 & 4.20 & 0.59 & 2.55 & - \\
\hline GC+MD & 49.20 & 20.95 & 8.71 & 4.94 & 5.02 & 3.77 & 2.92 & 2.91 & 1.10 & 0.47 & - & - \\
\hline RC+MD & 53.59 & 20.00 & 8.31 & 4.60 & 4.48 & 3.04 & 1.82 & 2.03 & 1.57 & 0.55 & - \\
\hline GC+MD+WT & 51.19 & 17.26 & 7.27 & 4.05 & 9.46 & 3.18 & 2.69 & 3.12 & 1.30 & 0.47 & - \\
\hline RC+MD+WT & 52.51 & 22.73 & 8.89 & 5.14 & 0.94 & 3.74 & 1.48 & 2.67 & 1.30 & 0.61 & - \\
\hline
\end{tabular}


Variations of the calculated total MAC values of the samples versus photon energies $(1 \mathrm{keV}-1 \mathrm{GeV})$ are shown in Fig. 1. In the low-energy range of $1-100 \mathrm{keV}$, the photoelectric process is predominant and total MAC values are directly affected by this process. It was seen that MAC values decreased sharply with increasing energy in this region. In the mid-energy range of $100 \mathrm{keV}-5 \mathrm{MeV}$, the Compton scattering (incoherent scattering) is dominant, and MAC values slightly changed in this region. At high energies, above $5 \mathrm{MeV}$, the Pair production process (nuclear field) starts, and an increase in MAC values was observed with increasing energy. As seen in Fig. 1, it can be noticed that the MAC values of the samples determined by both EpiXS and XCOM are in good agreement. This agreement is also seen obviously in Table 2 in the range of $1-200 \mathrm{keV}$ (above $200 \mathrm{keV}$, the values are almost the same). To make a detailed comparison about the shielding potentials of the samples, calculated MAC values of the clays were compared with those of other reported shielding materials and the data are given in Table 3. It can be said that the studied samples have more shielding abilities than the other given shielding materials. GC has lower MAC values than RC. GC reinforced with MD (GCMD) has higher MAC values than those of RC reinforced with MD (RCMD). After adding WT to the samples, it was observed that GC reinforced with MD and WT (GCMDWT) has higher MAC values than RC reinforced with MD and WT (RCMDWT). Among the reinforced samples, the best shielding capability is obtained for GCMDWT.

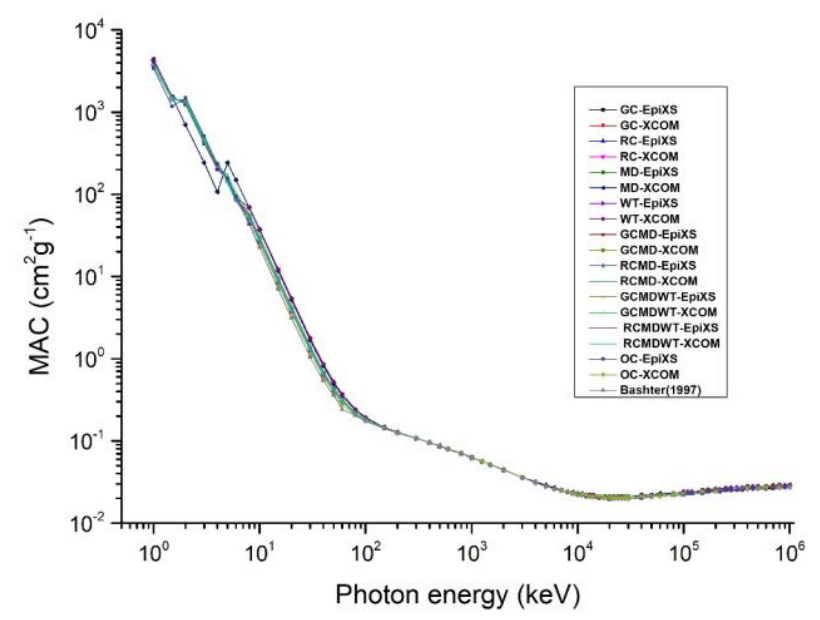

Figure 1. The changes of obtained MAC values of GC, RC, MD, WT, GCMD, RCMD, GCMDWT, RCMDWT and $\mathrm{OC}$ as a function of incident photon energies by EpiXS and XCOM.

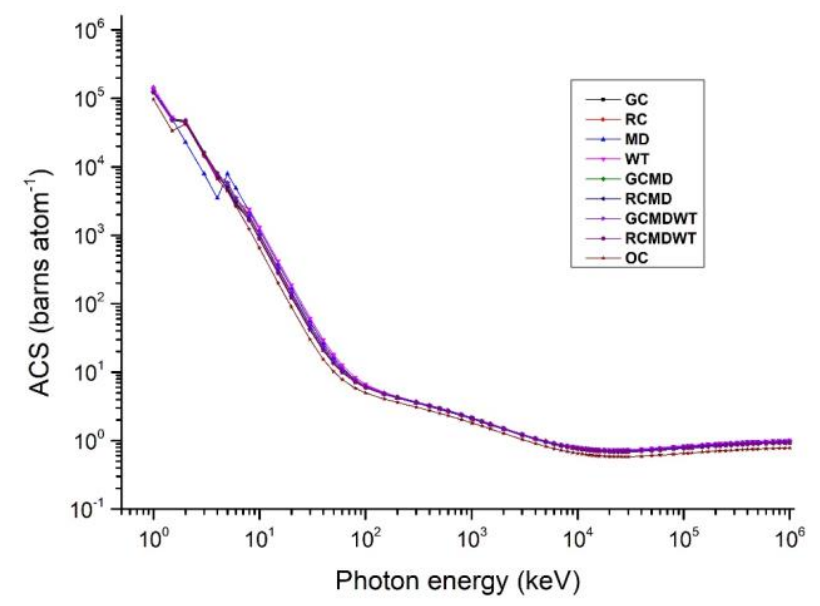

Figure 2. The changes of obtained ACS values of GC, RC, MD, WT, GCMD, RCMD, GCMDWT, RCMDWT and $\mathrm{OC}$ as a function of incident photon energies 
The changes of total atomic cross-sections versus incident photon energies are given in Fig. 2. The sample with higher ACS values can be defined as a better shielding material. RC has higher ACS values than GC in low, mid and high energy regions. By the addition of MD, GC show higher ACS values than RC. After being reinforced with WT, higher ACS values are still observed for the GC sample. Among the reinforced materials, it can be noticed that GCMDWT has more shielding ability than the others. ACS values of the samples were also compared with those of ordinary concrete (OC), a widely used shielding material [31]; it is observed that all the studied samples have higher protection features than OC.

Table 2. Obtained MAC values of the unreinforced and reinforced samples and ordinary concrete determined by EpiXS and XCOM in the energy range of 1-200keV

\begin{tabular}{|c|c|c|c|c|c|c|c|c|c|c|c|c|c|c|c|c|c|c|c|}
\hline nergy & Go & $\mathrm{C}$ & $\mathbf{R}$ & $\mathbf{2 C}$ & & ID & & NT & GCl & MD & $\mathbf{R C l}$ & MD & GCM & IDWT & RCM & IDWT & & OC & \\
\hline$(\mathrm{keV})$ & EpiXS & XCOM & EpiXS & XСОМ & EpiXS & XСОМ & EpiXS & XCOM & EpiXs & XCOM & EpiXS & XCOM & EpiXs & XСOM & EpiXS & XCOM & EpiXS & XCOM & [31] \\
\hline 1 & 30 & 30 & 3749 & 3748 & 4402 & 4403 & 4184 & 4182 & 3608 & 3608 & 3698 & 3698 & 765 & 765 & 602 & 602 & 3407 & - & - \\
\hline 1,5 & 71 & 1472 & 1439 & 0 & 7 & 8 & 3 & 4 & 5 & 6 & 4 & 5 & 0 & 1 & 17 & 18 & 1175 & - & - \\
\hline 2 & 17 & 19 & 1356 & 588 & 697.8 & 698.2 & 1242 & 1243 & 1384 & 1386 & 1335 & 7 & 271 & 1273 & 09 & 11 & 1489 & - & - \\
\hline 3 & 5.4 & 5.5 & 59.9 & 59.9 & 242.0 & 242.4 & 418.8 & 418.9 & 470.0 & 470.0 & 452.1 & 452.1 & 429.3 & 429.4 & 478.7 & 478.8 & 509.9 & - & - \\
\hline 4 & 2.5 & 2.6 & 0.1 & 0.2 & 6.8 & 7.4 & 2.6 & 2.8 & 6 & 8 & 0 & 1 & 9.4 & 6 & 3.7 & .8 & 2 & - & - \\
\hline 5 & 9 & 6.1 & 6.4 & 5 & .0 & .7 & 148.7 & 0 & 7 & .9 & 6 & 8 & .2 & 5 & .7 & 9 & 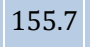 & - & - \\
\hline 6 & 87.86 & .85 & 88.09 & 88.09 & 149.1 & 149.1 & 89.88 & 89.91 & 94.47 & 94.46 & 87.64 & 87.63 & 103.7 & 103.7 & 81.95 & 81.94 & 93.67 & - & - \\
\hline 8 & 99 & .00 & 54.31 & .34 & 68.31 & 68.24 & 69.78 & .86 & 55.09 & 55.11 & 51.08 & 51.10 & 57.18 & 57.19 & 49.76 & 49.79 & 43.10 & - & - \\
\hline 10 & 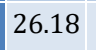 & 3 & 2 & 7 & 5 & 1 & 3 & 2 & 3 & 3 & 6 & 6 & 6 & 6 & 3 & 4 & 4 & . & 22.56 \\
\hline 15 & 79 & 71 & 85 & 276 & 11.69 & 67 & 12.25 & 24 & 370 & 360 & 679 & 8.670 & 9.734 & 9.722 & 8.446 & 8.439 & 7.063 & 150 & t \\
\hline 20 & 672 & 667 & 4.124 & 4.118 & 5.165 & 5.155 & 5.466 & 5.457 & 4.153 & 4.147 & 3.852 & 3.847 & 4.311 & 4.304 & 3.751 & 3.745 & 3.110 & 3.141 & 3.105 \\
\hline 30 & 229 & 228 & 1.372 & 1.370 & 1.684 & 1.680 & 1.796 & 1.792 & 1.378 & 1.375 & 1.287 & 1.285 & 25 & 1.423 & 56 & 1.254 & 1.049 & 1.056 & 1.048 \\
\hline 40 & 20 & 19 & 82 & 0.680 & 0.813 & 11 & 65 & 0 & 4 & 0 & 5 & 0. & 4 & 2 & 2 & 30 & 0.542 & 44 & 0 \\
\hline 50 & 98 & 97 & 30 & 29 & 8 & 96 & 5 & 24 & 1 & 0 & 1 & 0 & 41 & 40 & 04 & 4 & 9 & 9 & e \\
\hline 60 & 0.297 & 0.297 & 0.316 & 0.315 & 0.355 & 0.355 & 0.371 & 0.370 & 0.316 & 0.316 & 0.305 & 0.304 & 0.322 & 0.322 & 0.301 & 0.300 & 0.275 & 0.275 & 0.241 \\
\hline 80 & 212 & 212 & 0.220 & 0.220 & 0.237 & 0.237 & 0.243 & 0.243 & 0.220 & 0.220 & 215 & 0.215 & 0.223 & 0.222 & 0.214 & 0.213 & 0.204 & 0.204 & 0.204 \\
\hline 100 & 177 & 177 & 0.181 & 0.181 & 0.191 & 0.190 & 0.193 & 0.193 & 81 & 0.181 & 0 & 9 & 33 & 32 & 78 & 8 & 4 & 174 & 0.172 \\
\hline 150 & 142 & 142 & 143 & 0.143 & 0.147 & 0.147 & 0.146 & 0.146 & 0.143 & 0.143 & 0.142 & 0.142 & 0.143 & 0.144 & 0.142 & 0.142 & 0.142 & 0.142 & 0.14 \\
\hline 200 & 0.125 & 0.125 & 0.126 & 0.126 & 0.128 & 0.128 & 0.127 & 0.127 & 0.126 & 0.126 & 0.126 & 0.126 & 0.126 & 0.126 & 0.125 & 0.125 & 0.127 & 0.126 & 0.127 \\
\hline
\end{tabular}

The energy dependence of $Z_{\text {eff }}$ is given in Figs. 3-4. In the low energy region, due to the photoelectric effect, maximum $Z_{\text {eff }}$ values were obtained. By increasing energy, these values decreased sharply. Then the values gradually increased and remained constant in high energies. As can be seen from the figures, $Z_{\text {eff }}$ values of RC are higher than those of GC. By reinforced with MD, GC has higher $Z_{\text {eff }}$ values than RC. The addition of WT again kept GCMDWT showing its more shielding potential than the other reinforced materials.

Table 3. Obtained MAC values of the unreinforced and reinforced samples and other shielding materials

\begin{tabular}{|c|c|c|c|c|c|c|c|c|c|c|c|c|c|}
\hline $\begin{array}{c}\text { Energy } \\
(\text { (keV) }\end{array}$ & GC & RC & MD & WT & GCMD & RCMD & GCMDWT & RCMDWT & $\begin{array}{l}\text { Silica } \\
\text { Sand [6] }\end{array}$ & WI [20] & $\begin{array}{c}\text { Pumice } \\
\text { [20] }\end{array}$ & IO [22] & $\mathrm{OC}[31]$ \\
\hline 10 & 26.18 & 29.16 & 36.75 & 37.91 & 29.53 & 27.36 & 30.66 & 26.63 & 19.88 & 26.09 & 25.01 & 23.40 & 22.74 \\
\hline 15 & 8.279 & 9.285 & 11.69 & 12.25 & 9.370 & 8.679 & 9.734 & 8.446 & 6,110 & 8.215 & 7.846 & 7.439 & 7.063 \\
\hline 20 & 3.672 & 4.124 & 5.165 & 5.466 & 4.153 & 3.852 & 4.311 & 3.751 & 2,685 & 3.635 & 3.468 & 3.265 & 3.110 \\
\hline 30 & 1.229 & 1.372 & 1.684 & 1.796 & 1.378 & 1.287 & 1.425 & 1.256 & 0.916 & 1.215 & 1.162 & 1.094 & 1.049 \\
\hline 40 & 0.620 & 0.682 & 0.813 & 0.865 & 0.684 & 0.645 & 0.704 & 0.632 & 0.484 & 0.614 & 0.591 & 0.560 & 0.542 \\
\hline 50 & 0.398 & 0.430 & 0.498 & 0.525 & 0.431 & 0.411 & 0.441 & 0.404 & 0.328 & 0.395 & 0.383 & 0.367 & 0.359 \\
\hline 60 & 0.297 & 0.316 & 0.355 & 0.371 & 0.316 & 0.305 & 0.322 & 0.301 & 0.257 & 0.295 & 0.288 & 0.279 & 0.275 \\
\hline 80 & 0.212 & 0.220 & 0.237 & 0.243 & 0.220 & 0.215 & 0.223 & 0.214 & 0.195 & 0.211 & 0.208 & 0.204 & 0.204 \\
\hline 100 & 0.177 & 0.181 & 0.191 & 0.193 & 0.181 & 0.179 & 0.183 & 0.178 & 0.169 & 0.177 & 0.175 & 0.182 & 0.174 \\
\hline 150 & 0.142 & 0.143 & 0.147 & 0.146 & 0.143 & 0.142 & 0.143 & 0.142 & 0.140 & 0.142 & 0.141 & 0.143 & 0.142 \\
\hline
\end{tabular}



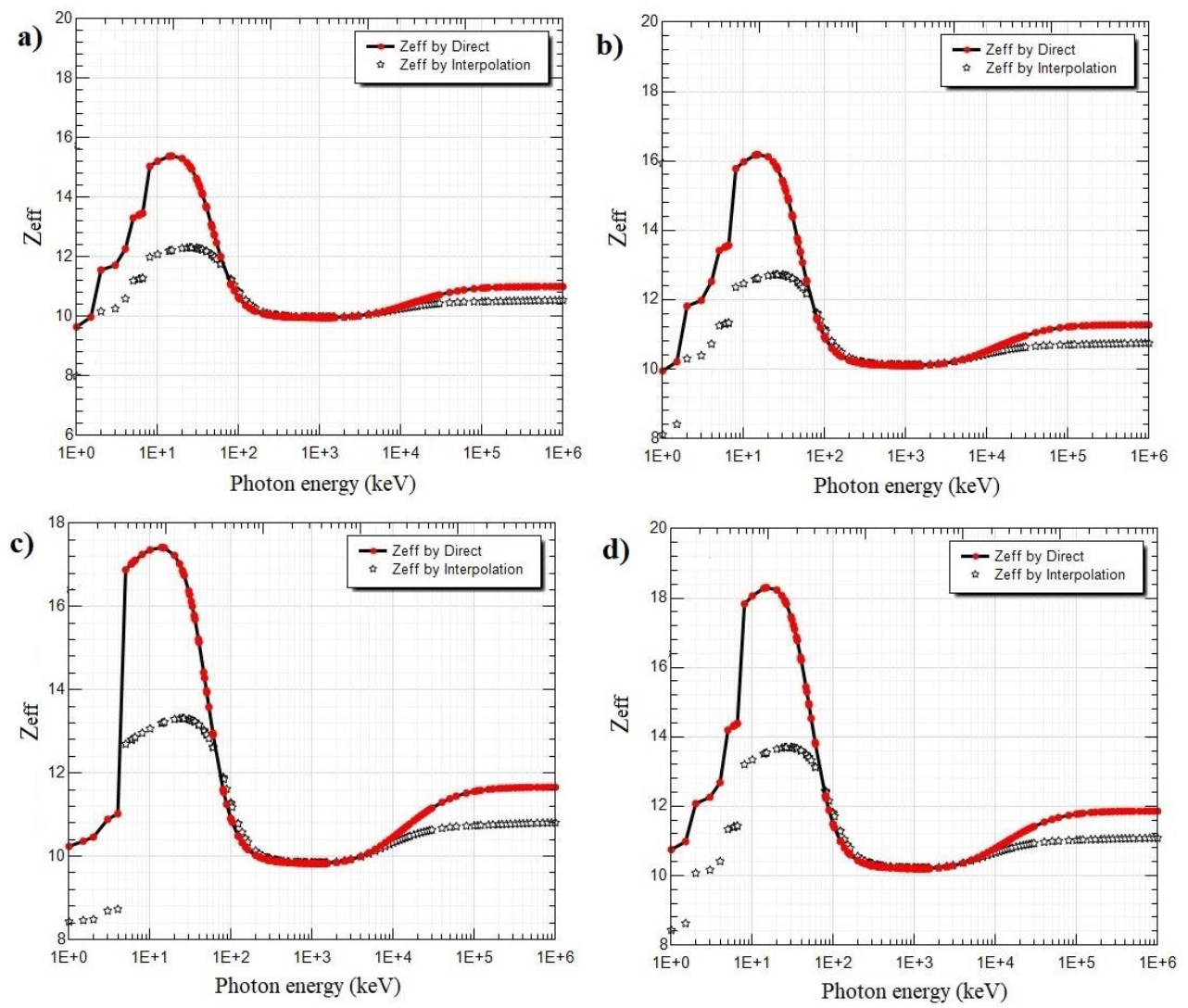

Figure 3. The changes of $Z_{\text {eff }}$ values of GC (a) RC (b) MD (c) WT (d) as a function of incident photon energies.

Variation of $N_{\text {eff }}$ values versus photon energies is shown in Figs. 5-6. $N_{\text {eff }}$ is one of the most critical parameters that represents the effective conductivity of the compound depending on the excitatory photon energy. As seen in the figures, the variation of $N_{\text {eff }}$ values versus incident photon energies is similar to that of $Z_{\text {eff }}$ values. The interactions between photons and material with the photoelectric effect, Compton scattering, and pair production processes cause changes in the number of free electrons in the material.

EABF and EBF of the samples were calculated in 1-40 mfp depth range in the photon energy range of 0.015-15MeV. The changes of EABF and EBF versus incident photon energies were given in Figs. 7-10. As low-energy photons are absorbed by their all energies due to the photoelectric effect, buildup factor values are small in low photon energies. Compton scattering is the dominant effect in the mid-energy region, so the photons are not completely disappearing; only the energy decreases in this process. As a result, a large number of scattered photons are observed, and this causes an increase in the accumulation of photons. Thus, EABF and EBF values reach great values at medium energies. The dominant effect in high energy region is pair production, and this causes a strong absorption of photons. Therefore, the buildup factors again decrease in the high energy region [32]. As seen in Figs. 7-10, in the 1-40 mfp depth region of the samples, the buildup factors increase with increasing penetration depth. The maximum values of the buildup factors were obtained at $40 \mathrm{mfp}$.

It was observed that the buildup factors change significantly with the change of photon energies, depth of penetration and different chemical compositions of the samples. According to the obtained values of EABF and EBF, it can be mentioned that the photons cluster slightly more for GC than RC before reinforcing. By the addition of MD, RCMD has higher EABF and EBF values than GCMD. After WT addition, the RCMDWT sample gives higher EABF and EBF values. Among all samples, the highest buildup factors are observed for the GC sample. Therefore, it can be said that the maximum Compton scattering effect is observed for GC. 

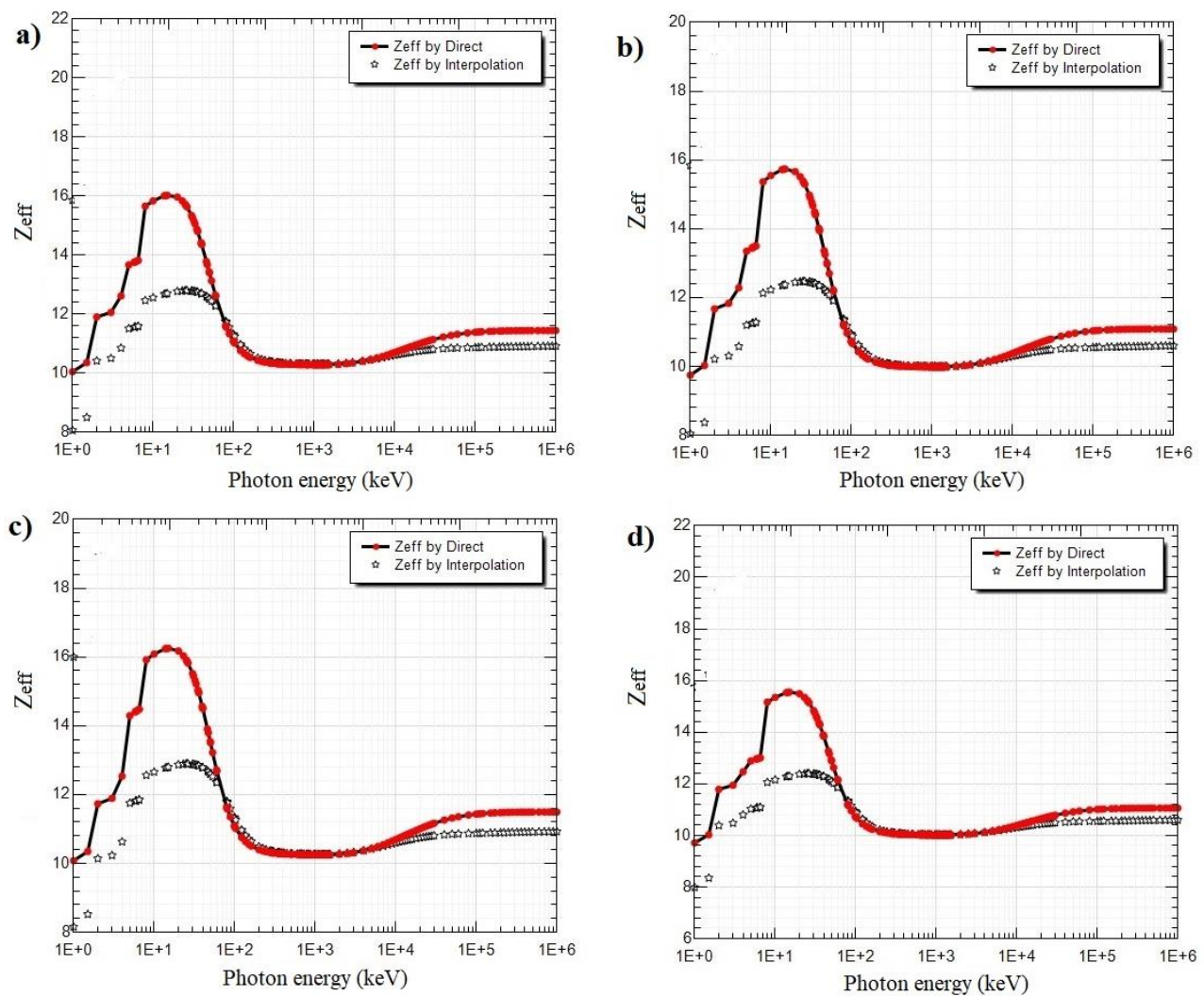

Figure 4. The changes of $Z_{\text {eff }}$ values of GCMD (a) RCMD (b) GCMDWT (c) RCMDWT (d) as a function of incident photon energies.
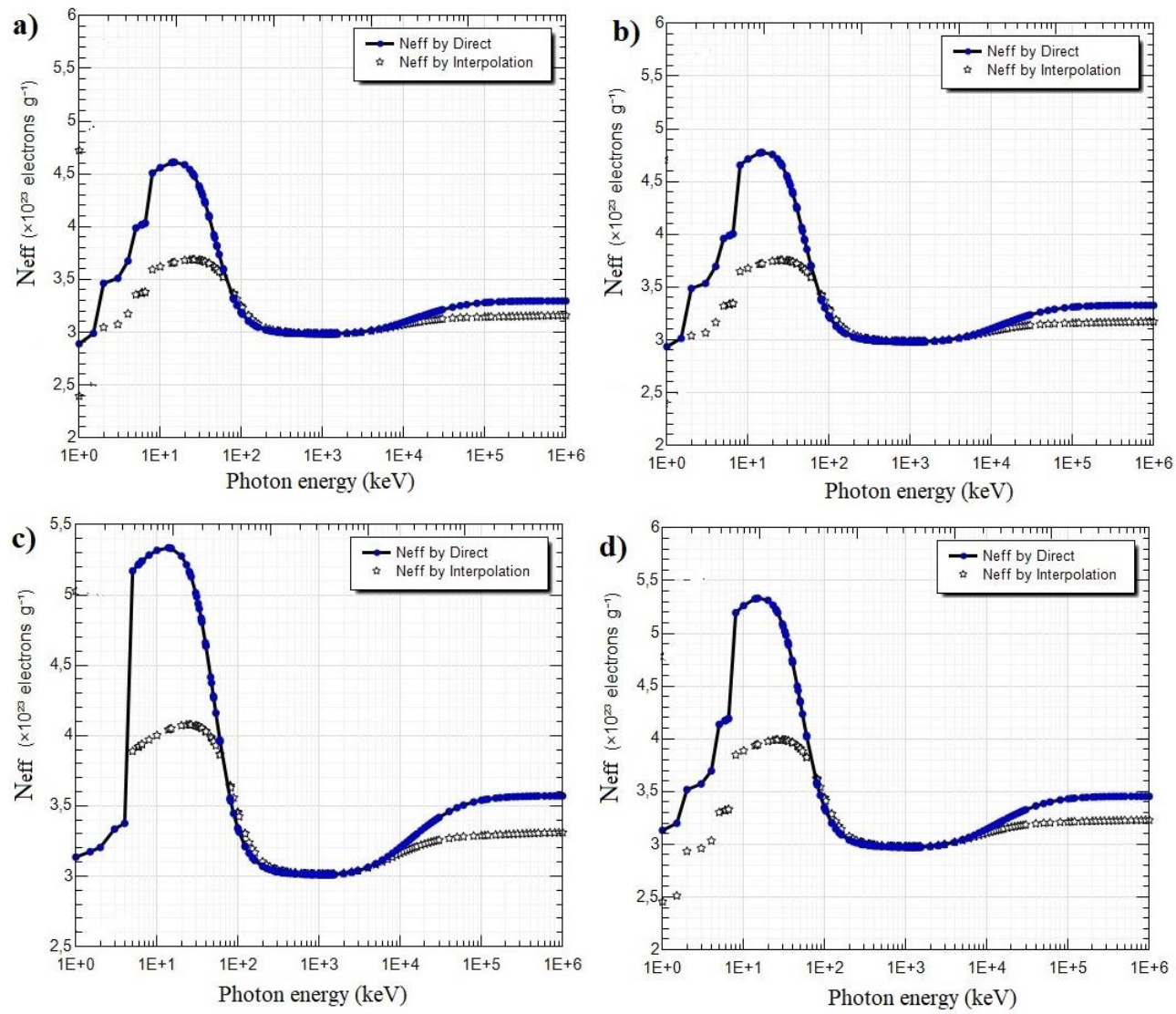

Figure 5. The variations of $N_{\text {eff }}$ values of GC (a) RC (b) MD (c) WT (d) versus incident photon energies. 
Equivalent atomic number $\left(Z_{e q}\right)$ is the parameters that correspond to the interaction between radiation and matter. $Z_{e q}$ is evaluated for the determination of absorbed dose, buildup factor, and energy absorption calculation. While $Z_{\text {eff }}$ is calculated by adding all partial photon interaction processes, Compton scattering is the main process in determining $Z_{e q}$ [33]. The obtained $Z_{e q}$ values of the samples are listed in Table 4. As mentioned above, for $Z_{\text {eff }}, Z_{e q}$ of RC is higher than GC, while GCMD has higher $Z_{e q}$ values than RCMD. $Z_{e q}$ values of RCMDWT are still lower than those of GCMDWT after WT addition.
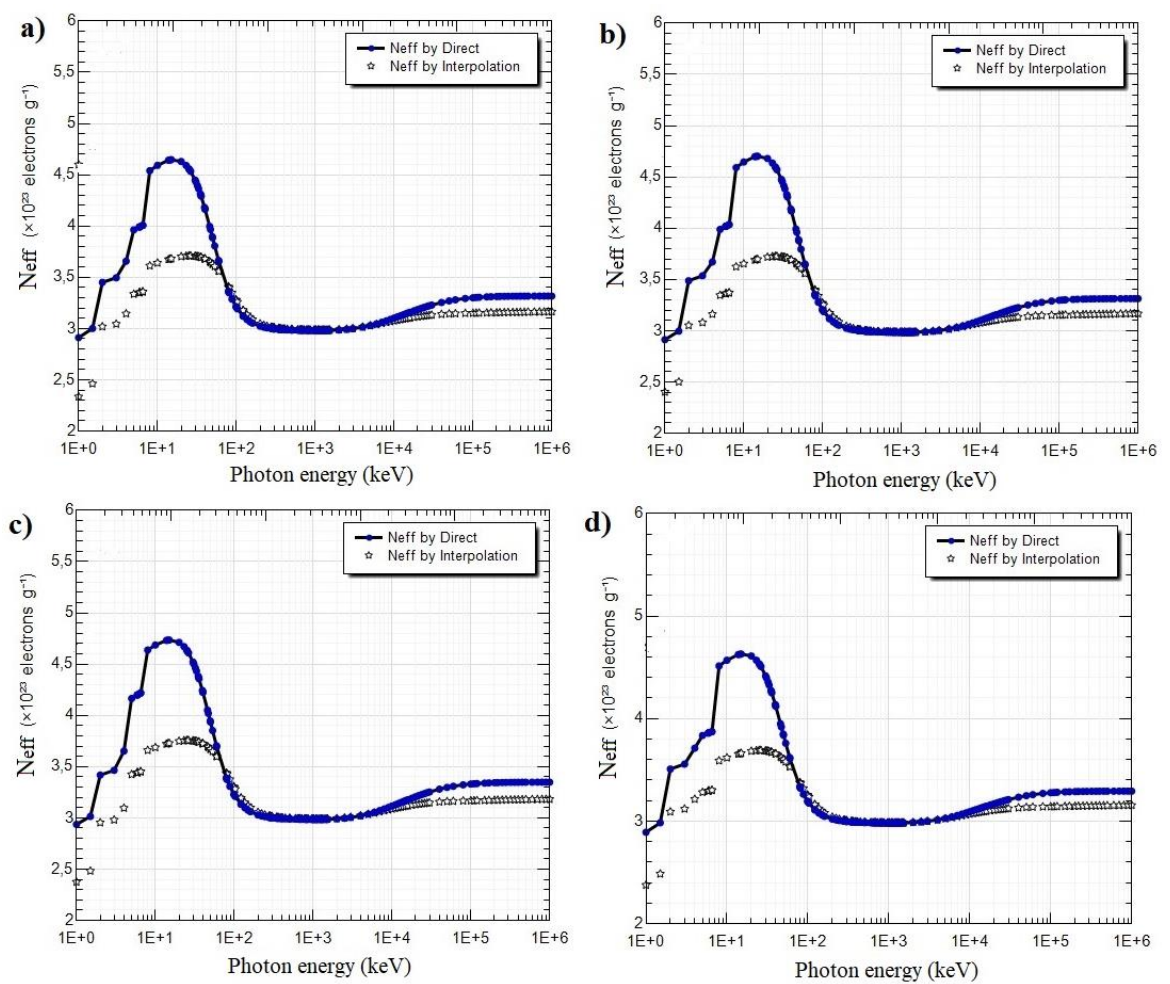

Figure 6. The variations of $N_{\text {eff }}$ values of GCMD (a) RCMD (b) GCMDWT (c) RCMDWT (d) versus incident photon energies.
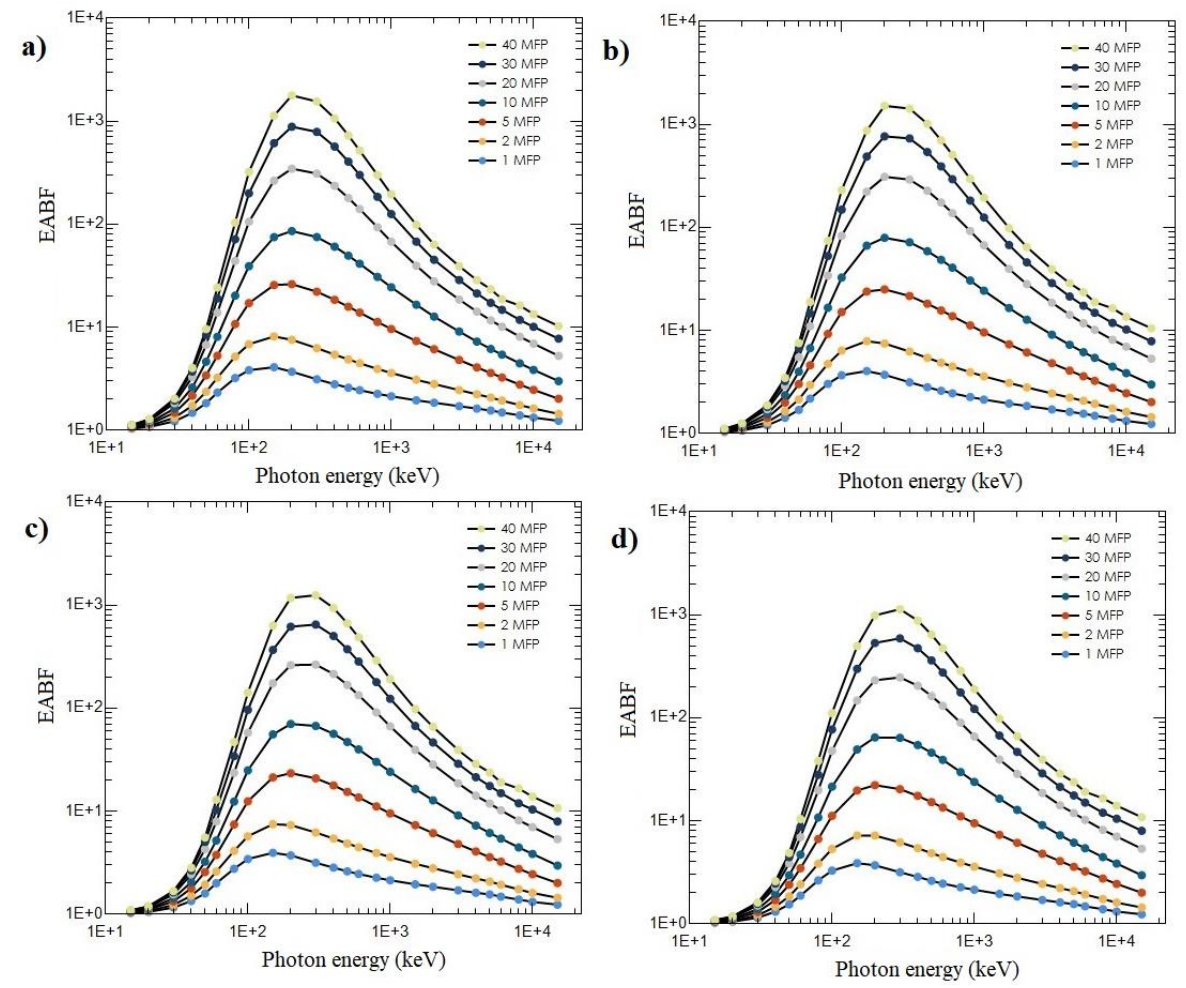

Figure 7. The changes of EABF of GC (a) RC (b) MD (c) WT (d) versus incident photon energies. 

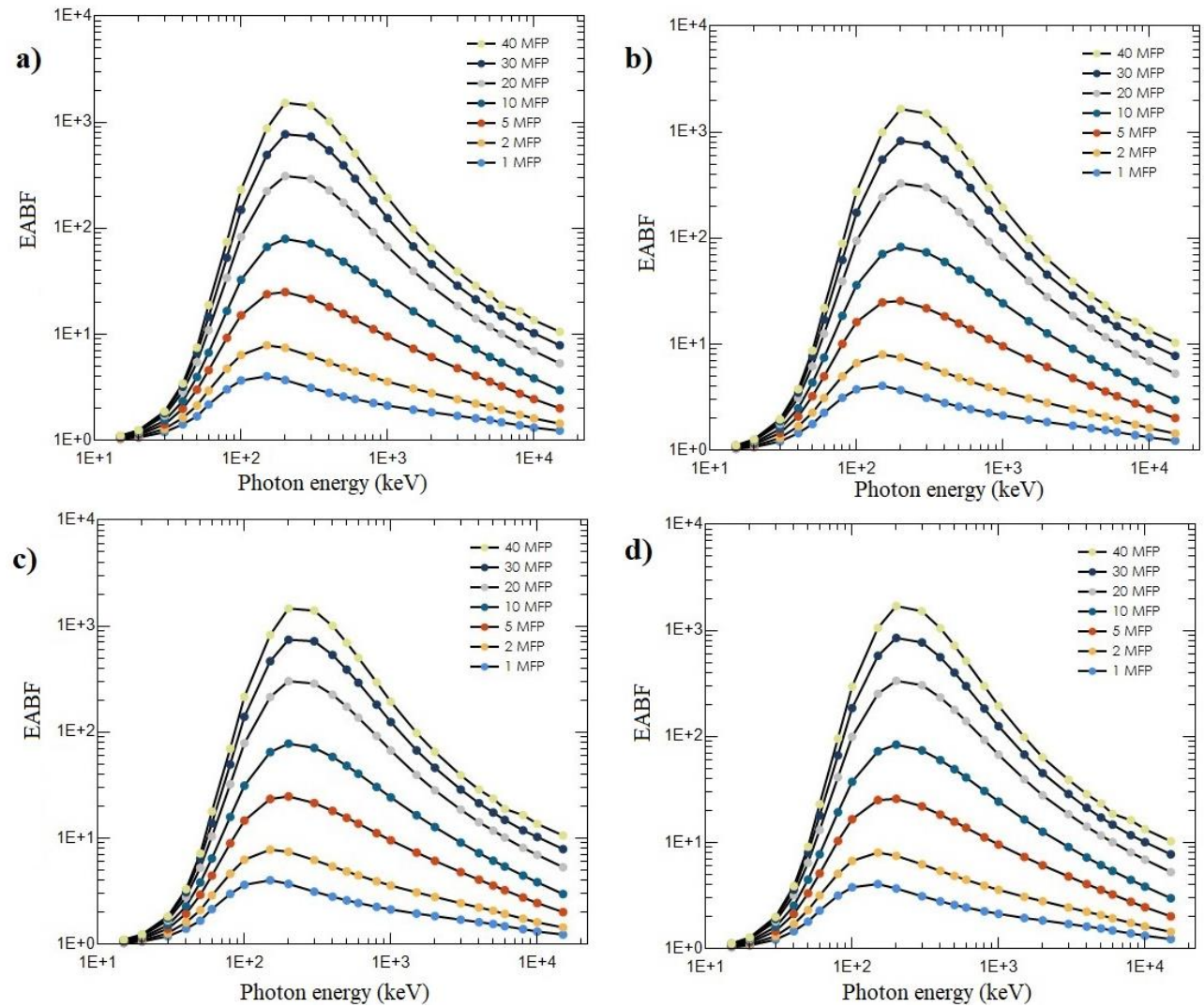

Figure 8. The changes of EABF of GCMD (a) RCMD (b) GCMDWT (c) RCMDWT (d) versus incident photon energies.
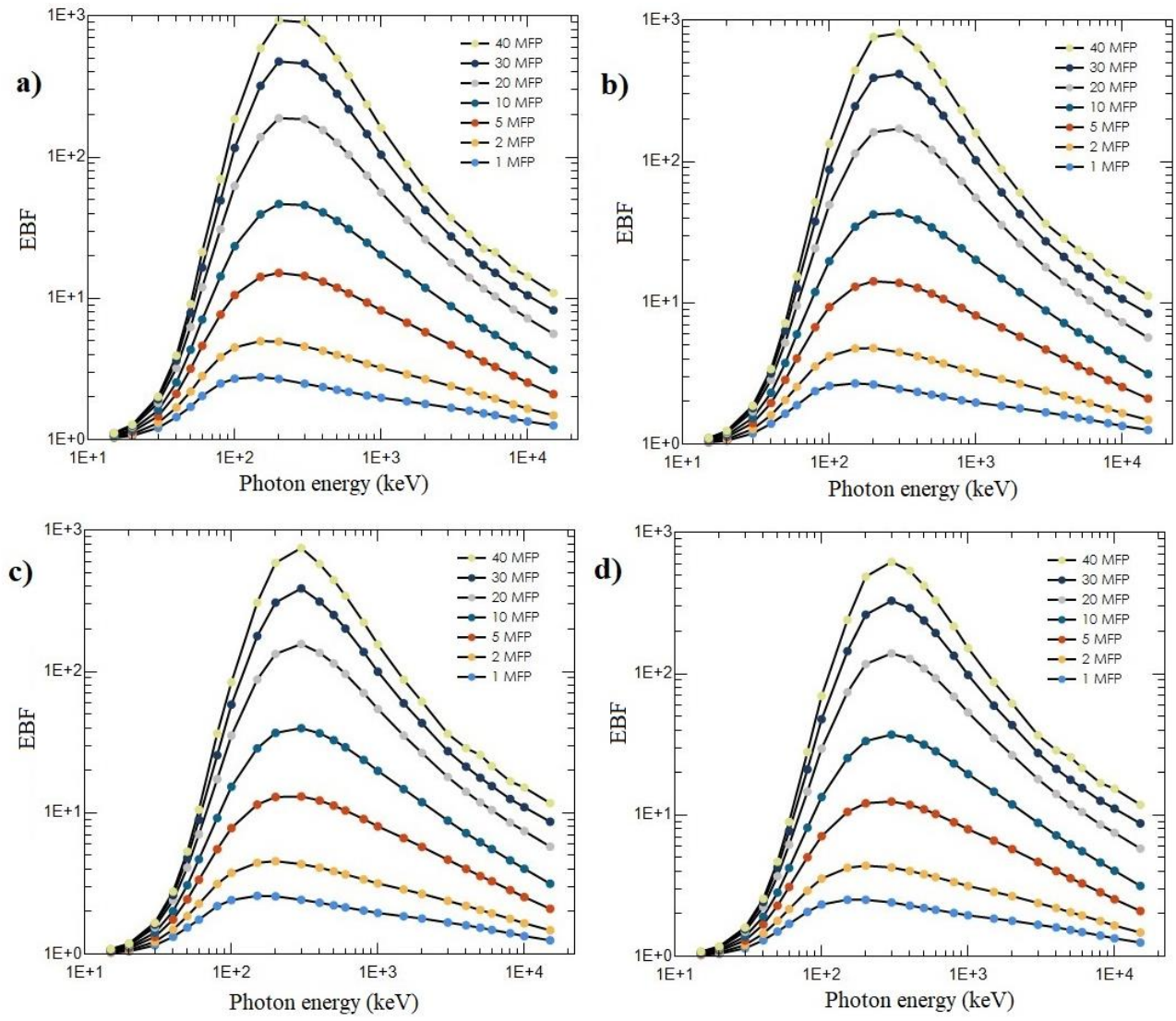

Figure 9. The variations of EBF of GC (a) RC (b) MD (c) WT (d) as a function of incident photon energies. 

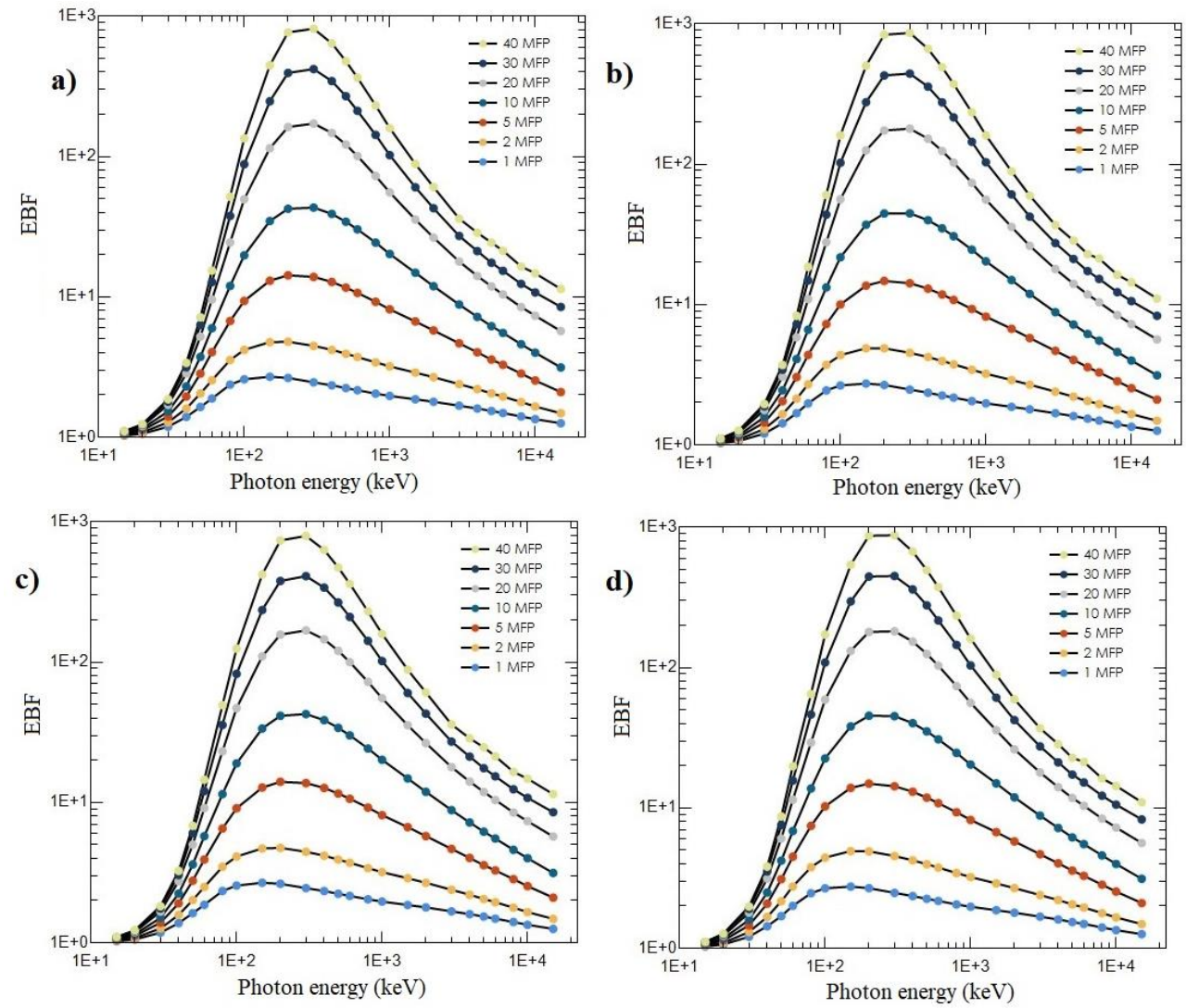

Figure 10. The variations of EBF of GCMD (a) RCMD (b) GCMDWT (c) RCMDWT (d) as a function of incident photon energies.

\section{Conclusions}

The present paper determined the radiation-matter interaction parameters of unreinforced and reinforced GC and RC obtained from Oltu/Erzurum in Turkey. For this purpose, we used EpiXS software to calculate the photon attenuation parameters, MAC, ACS, $Z_{\text {eff }} N_{\text {eff }}$ buildup factors and $Z_{\text {eq. }}$ MAC values of the studied samples were also calculated by XCOM software to validate the determined EpiXS results. A good agreement was obtained between the values. According to the obtained results, we can conclude that RC has more shielding features than GC. However, GCMDWT show more shielding ability than RCMDWT among reinforced samples. This result indicates that adding MD and WT increase the shielding feature of the GC. It is clear that the increase of Ca content in reinforced GC improves the shielding potential of the sample. Therefore, it can be said that chemical composition is one of the factors which affect the shielding property of the materials. Among all the studied clayey soil samples, GCMDWT has the highest shielding capability. It can be said that the maximum Compton scattering effect is observed for GC due to the obtained highest buildup factors. As a result of comparing MAC and ACS values of the samples with ordinary concrete, we can conclude that all the studied samples have more shielding potential than a widely preferred shielding material, ordinary concrete. Therefore, the studied clayey (both unreinforced and reinforced) materials can be used as building materials for shielding purposes in many places related to radiation. 
Table 4. $Z_{e q}$ values of the unreinforced and reinforced samples determined by EpiXS.

\begin{tabular}{|c|c|c|c|c|c|c|c|c|}
\hline $\begin{array}{c}\text { Energy } \\
(\mathrm{keV})\end{array}$ & GC & $\mathrm{RC}$ & M & W & GCM & RCM & GCMW & RCMW \\
\hline 15 & 12.89694 & 13.37959 & 14.31205 & 14.60366 & 13.42975 & 13.08630 & 13.58021 & 12.98012 \\
\hline 20 & 13.05550 & 13.56008 & 14.50558 & 14.83670 & 13.59793 & 13.26079 & 13.74937 & 13.15151 \\
\hline 30 & 13.23567 & 13.75602 & 14.69286 & 15.08732 & 13.77945 & 13.44850 & 13.92991 & 13.33993 \\
\hline 40 & 13.35366 & 13.88703 & 14.81830 & 15.25659 & 13.90104 & 13.57192 & 14.05380 & 13.46337 \\
\hline 50 & 13.43449 & 13.98435 & 14.91022 & 15.37267 & 13.99203 & 13.65885 & 14.14408 & 13.54863 \\
\hline 60 & 13.49492 & 14.05589 & 14.98193 & 15.46018 & 14.05823 & 13.72450 & 14.20851 & 13.61318 \\
\hline 80 & 13.58402 & 14.15710 & 15.07118 & 15.58434 & 14.15203 & 13.82381 & 14.29917 & 13.71033 \\
\hline 100 & 13.64202 & 14.22010 & 15.12996 & 15.66524 & 14.21049 & 13.88902 & 14.35745 & 13.77365 \\
\hline 150 & 13.73860 & 14.32201 & 15.20895 & 15.79824 & 14.30425 & 13.99865 & 14.44930 & 13.88156 \\
\hline 200 & 13.79929 & 14.38330 & 15.25712 & 15.87674 & 14.36087 & 14.05458 & 14.50563 & 13.94790 \\
\hline 300 & 13.87079 & 14.45799 & 15.31306 & 15.97470 & 14.42956 & 14.12087 & 14.57360 & 14.02296 \\
\hline 400 & 13.91459 & 14.49992 & 15.34185 & 16.02170 & 14.46838 & 14.16017 & 14.61134 & 14.06281 \\
\hline 500 & 13.93404 & 14.52353 & 15.36084 & 16.04983 & 14.48993 & 14.17899 & 14.63327 & 14.08116 \\
\hline 600 & 13.94705 & 14.53786 & 15.36967 & 16.06470 & 14.50298 & 14.19129 & 14.64596 & 14.09362 \\
\hline 800 & 13.95293 & 14.54443 & 15.37210 & 16.07471 & 14.50861 & 14.19672 & 14.65102 & 14.09938 \\
\hline 1000 & 13.96343 & 14.55146 & 15.37628 & 16.07892 & 14.51544 & 14.20494 & 14.65731 & 14.10801 \\
\hline 1500 & 11.94591 & 12.38245 & 13.10451 & 13.49454 & 12.48925 & 12.10903 & 12.59738 & 12.04416 \\
\hline 2000 & 11.44070 & 11.78642 & 12.40171 & 12.61042 & 11.93270 & 11.56173 & 12.02207 & 11.51112 \\
\hline 3000 & 11.31237 & 11.63247 & 12.19695 & 12.37299 & 11.78646 & 11.42222 & 11.86702 & 11.37830 \\
\hline 4000 & 11.27695 & 11.59001 & 12.14171 & 12.30958 & 11.74566 & 11.38376 & 11.82390 & 11.34142 \\
\hline 5000 & 11.26036 & 11.57239 & 2.11927 & 12.28483 & 11.72984 & 11.36658 & 11.80725 & 11.32484 \\
\hline 6000 & 11.25366 & 11.56218 & 12.10295 & 12.26577 & 11.71913 & 11.35857 & 11.79549 & 11.31743 \\
\hline 8000 & 11.24021 & 11.54825 & 12.08636 & 12.24656 & 11.70645 & 11.34481 & 11.78233 & 11.30388 \\
\hline 10000 & 11.23472 & 11.54181 & 12.07684 & 12.23664 & 11.70026 & 11.33895 & 11.77559 & 11.29831 \\
\hline 15000 & 11.22824 & 11.53492 & 12.06371 & 12.22419 & 11.69371 & 11.33228 & 11.76818 & 11.29210 \\
\hline
\end{tabular}

\section{Author Contributions}

All authors contributed equally to this work. They all read and approved the last version of the manuscript.

\section{Conflicts of Interest}

The authors declare no conflict of interest. 


\section{References}

[1] Z. Aygun, N. Yarbasi, A Spectroscopic Analysis of Clay Types and Silty Sand from Oltu/Erzurum (Turkey) Region Reinforcing with Marble Dust and Waste Tire, Karaelmas Science and Engineering Journal, 9(2), (2019) 215-226.

[2] M. Jafari, M. Esnaashari, Effect of waste tire cord reinforcement on unconfined compressive strength of lime stabilized clayey soil under freeze-thaw condition, Cold Regions Science and Technology, 82, (2012) 21-29.

[3] R. T. Erdem, A. U. Oztürk, Effect of Marble Powder Additive on Freezing-Thawing Properties of Cement Mortar, BEU Journal of Science, 1(2), (2012) 85-91.

[4] N. Yarbasi, E. Kalkan, The Mechanical Performance of Clayey Soils Reinforced with Waste PET Fibers, International Journal of Earth Science and Knowledge Applications, 2(1), (2020) 19-26.

[5] B. Aygün, Neutron and gamma radiation shielding properties of high-temperature-resistant heavy concretes including chromite and wolframite, Journal of Radiation Research and Applied Sciences, 12, (2019) 352-359.

[6] R. S. M. Rashid, S. M. Salem, N. M. Azreen, Y. L. Voo, M. Haniza, A. A. Shukri, M. S. Yahya, Effect of elevated temperature to radiation shielding of ultra-high-performance concrete with silica sand or magnetite, Construction and Building Materials, 262, (2020) 120567

[7] O. Gencel, A. Bozkurt, E. Kam, T. Korkut, Determination and calculation of gamma and neutron shielding characteristics of concretes containing different hematite proportions, Annals of Nuclear Energy, 38, (2011) 2719-2723.

[8] A. Gür, B. Artıg, T. Cakır, Photon attenuation properties of concretes containing magnetite and limonite ores, Physicochemical Problems of Mineral Process, 53(1), (2017) 184-191.

[9] I. Akkurt, H. Canakci, Radiation attenuation of boron-doped clay for 662, 1173 and 1332 keV gamma rays, Iranian Journal of Radiation Research, 9(1), (2011) 37-40.

[10] H. S. Mann, G. S. Brar, G. S. Mudahar, Gamma-ray shielding effectiveness of novel light-weight clayflyash bricks, Radiation Physics and Chemistry, 127, (2016) 97-101.

[11] S. F. Olukotun, S. T. Gbenu, F. I. Ibitoye, O. F. Oladejo, H. O. Shittu, M. K. Fasasi, F. A. Balogun, Investigation of gamma radiation shielding capability of two clay materials, Nuclear Engineering and Technology, 50, (2018) 957-962.

[12] M. J. Berger, J. H. Hubbell, XCOM: Photon Cross Sections Database, Web Version 1.2. National Institute of Standards and Technology Gaithersburg, MD, (1987) 20899, USA, available at. http://physics.nist.gov/xcom.

[13] S. Agostinelli, J. Allison, K. Amako, J. Apostolakis, H. Araujo, et al., Geant4-a simulation toolkit, Nuclear Instrumentation and Methods Physics Research Section A, 506, (2003) 250-303.

[14] L. Gerward, N. Guilbert, K. B. Jensen, H. Levring, X-ray absorption in matter. Reengineering XCOM, Radiation Physics and Chemistry, 60, (2001) 23-24.

[15] L. Gerward, N. Guilbert, K. B. Jensen, H. Levring, WinXCom-a program for calculating X-ray attenuation coefficients, Radiation Physics and Chemistry, 71, (2004) 653-654.

[16] R. Nowotny, XMuDat: Photon attenuation data on PC, IAEA Report IAEA-NDS, (1998) 195.

[17] E. Sakar, Ö. F. Özpolat, B. Alım, M.I. Sayyed, M. Kurudirek, Phy-X / PSD: Development of a user friendly online software for calculation of parameters relevant to radiation shielding and dosimetry, Radiation Physics and Chemistry, 166, (2020) 1-12.

[18] K. S. Mann, S. S. Mann, Py-MLBUF: Development of an online-platform for gamma-ray shielding calculations and investigations, Annals of Nuclear Energy, 150, (2021) 107845.

[19] F. C. Hila, A. A. Astronomo, C. A. M. Dingle, J. F. M. Jecong, A. M. V. Javier-Hila, et al., EpiXS: A Windowsbased program for photon attenuation, dosimetry and shielding based on EPICS2017 (ENDF/BVIII) and EPDL97 (ENDF/B-VI8), Radiation Physics and Chemistry, 182, (2021) 109331. 
[20]Z. Aygun, M. Aygun, A study on usability of Ahlat ignimbrites and pumice as radiation shielding materials, by using EpiXS code, International Journal of Environmental Science and Technology, (2021). https://doi.org/10.1007/s13762-021-03530-9.

[21] M. B. Z. Gili, F. C. Hila, Investigation of Gamma-ray Shielding Features of Several Clay Materials Using the EPICS2017 Library, Philippine Journal of Science, 150 (5), (2021) 1017-1026.

[22] Z. Aygun, N. Yarbasi, M. Aygun Spectroscopic and radiation shielding features of Nemrut, Pasinler, Sarıkamıs and Ikizdere obsidians in Turkey: Experimental and theoretical study, Ceramics International, (2021). https://doi.org/10.1016/j.ceramint.2021.08.330.

[23] A. H. Almuqrin, M. I. Sayyed, J. F. M. Jecong, A. Kumar, M. M. AlShammari, B. Albarzan, SrO-SiO2B203-ZrO2 glass system: Effects of varying SrO and BaO compositions to physical and optical properties, and radiation shielding using EPDL2017 photoatomic library, Optik, 245, (2021) 167670.

[24] N. Yarbasi, Geotechnical mapping of Oltu (Erzurum) residential area and its vicinity, Pamukkale University Journal of Engineering and Science, 22, (2016) 538-545.

[25] D. F. Jackson, D.J. Hawkes, $X$-ray attenuation coefficients of elements and mixtures, Physics Reports, 70, (1981) 169-233.

[26] L. F. Pires, Soil analysis using nuclear techniques: a literature review of the gamma ray attenuation method, Soil Tillage Research, 184, (2018) 216-234.

[27] S. Prabhu, A. C. Sneha, P. P. Shetty, A. A. Narkar, S. G. Bubbly, S. B. Gudennavar, Effective atomic number and electron density of some biologically important lipids for electron, proton, alpha particle and photon interactions, Applied Radiation and Isotopes, 160, (2020) 109137.

[28] Y. Harima, An historical review and current status of buildup factor calculations and applications, Radiation Physics and Chemistry, 41(4-5), (1993) 631-672.

[29] Y. Harima, Y. Sakamoto, S. Tanaka, M. Kawai, Validity of the geometric-progression formula in approximating gamma-ray buildup factors, Nuclear Science and Engineering, 94(1), (1986) 24-35.

[30] ANSI/ANS 643, Gamma-ray Attenuation Coefficients and Buildup Factors for Engineering Materials, American Nuclear Society La Grange Park Illinois (1991).

[31] I. I. Bashter, Calculation of radiation attenuation coefficients for shielding concretes, Annals of Nuclear Energy, 24(17), (1997) 1389-1401.

[32] N. Kücük, O. Gezer, Determination of Build-up Factors for Natural Black Obsidian Ores, AKU Journal of Science and Engineering, 17, (2017) 872-880.

[33] S. S. Obaid, M. I. Sayyed, D. K. Gaikwad, P. P. Pawara, Attenuation coefficients and exposure buildup factor of some rocks for gamma ray shielding applications, Radiation Physics and Chemistry, 148, (2018) 86-94. 